\title{
TIC/TAC y COVID-19: uso y necesidades del profesorado de se- cundaria en Galicia
}

\section{Milagros Torrado Cespón}

milagros.torrado@unir.net

Universidad Internacional de la Rioja, España

\begin{abstract}
Resumen
La brecha digital en Galicia durante el confinamiento debido a la COVID-19 ha mostrado las necesidades del profesorado para hacer un uso eficiente de las TIC/TAC. El objetivo de este estudio es analizar las dificultades en el uso de las TIC/TAC con una muestra de 201 profesores de secundaria y proponer soluciones a éstas. Los sujetos realizaron un cuestionario online sobre el uso de estas herramientas antes y durante el confinamiento y los obstáculos encontrados para su aplicación. Además, un espacio de opinión libre aporta precisión al análisis cuantitativo. Los resultados muestran como la brecha digital es un problema tanto para alumnos como para profesores, evidenciándose, no solo la falta de equipamiento, sino también la falta de preparación. Formación continua de calidad y ayudas institucionales para la reducir el impacto socioeconómico parecen ser la solución. Se sugiere un cambio en las metodologías de enseñanza para proporcionar a los profesores un mejor entendimiento de la generación $Z$.
\end{abstract}

\section{Palabras clave}

Brecha digital; educación secundaria; TIC/TAC; COVID-19; generación Z. 


\title{
ICT/LKT and COVID-19: Use and needs of Galician secondary teachers
}

\author{
Milagros Torrado Cespón \\ milagros.torrado@unir.net \\ Universidad Internacional de la Rioja, España
}

\begin{abstract}
The digital divide Galicia during COVID-19 lockdown showed the need of offering teachers better resources for efficient use of ICT/LKT both in and outside the classroom. This research aims at analysing the difficulties regarding the use of ICT/LKT in a sample of 201 secondary school teachers and providing solutions for these. Teachers answered an online questionnaire about the use of these tools before and during the lockdown and the obstacles found in their application. Moreover, a free comment section complements the quantitative data analysis. Results show how the digital divide is a major issue both for teachers and students. It is not just a problem of resources, but also lack of training. Quality continuous training and help from institutions to lower the socioeconomic impact of the digital divide seem to be the best solution. A methodological change to provide teachers with a better understanding of Generation $\mathrm{Z}$ is also suggested.
\end{abstract}

\section{Key words}

Digital divide; secondary education; ICT/LKT; COVID-19; Generation Z. 


\section{Introducción}

En 2005, Dewan y Riggins (2005) apuntaban al problema y preocupación sobre la brecha digital que surgió durante la década de 1990. Más de treinta y quince años después, respectivamente, esta preocupación ha demostrado ser todavía una realidad. En un mundo occidental que se jacta de la preparación y la hiper conexión, la cruda realidad ha golpeado a muchas personas cuando el teletrabajo se convirtió en una necesidad debido periodo de confinamiento y posterior nueva normalidad causados por la expansión pandémica de COVID-19. En 2020, occidente se dio cuenta de que la brecha digital todavía estaba allí. España es uno de esos países que vio cómo la falta de infraestructura y la falta de recursos afectaron a muchas familias durante este período donde el problema no era sólo tener una conexión mejor o peor, sino también la situación económica y la posibilidad de utilizar un ordenador o un dispositivo similar para algo que no estaba relacionado con el ocio. Estas limitaciones afectaron a varias esferas, pero este documento se centra en la enseñanza, que, al mismo tiempo, nos lleva a la exclusión social (Castells, 2000a; Castells, 2000b; Sparks, 2013).

Aunque hay miles de publicaciones sobre los beneficios del uso de las tecnologías de la información y la comunicación (TIC) en la educación y, más concretamente, de las tecnologías de aprendizaje y conocimiento (TAC), lo cierto es que la calidad y la motivación en la educación en línea es difícil de lograr si la brecha digital sigue aquí y los profesores carecen de recursos y formación. Esta situación ha afectado en mayor o menor medida a diferentes zonas de España. En este caso, esta investigación ha analizado la experiencia de los profesores de secundaria gallegos. Galicia (noroeste de España) sigue en el proceso de la plena implantación de internet de alta velocidad (fig. 1), siendo las zonas rurales las más afectadas, pero también ciudades como su capital, Santiago de Compostela, donde las infraestructuras y el patrimonio a veces son difíciles de combinar sin daños y la banda ancha fija se retrasó hasta 2020 (Mosteiro, 2020) pero no se había hecho realidad en el momento en que se llevó a cabo esta investigación.

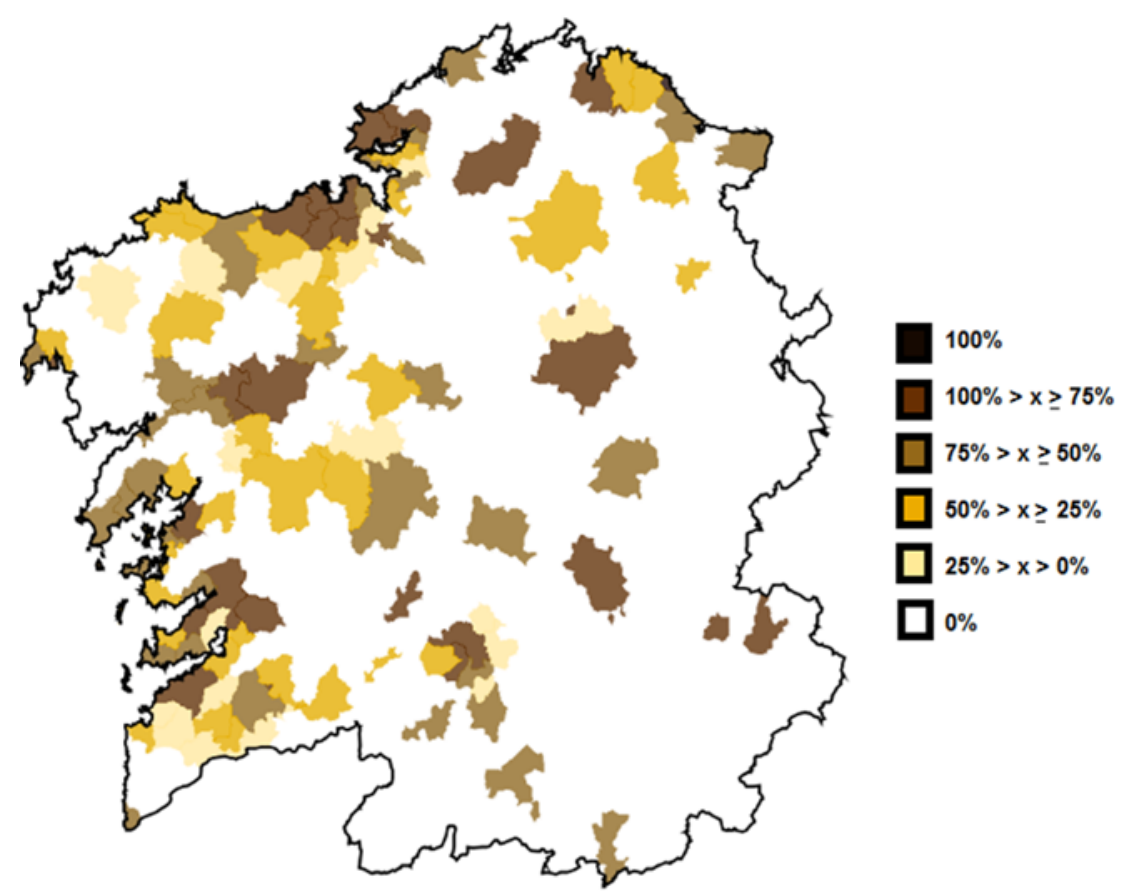

Figura 1. Disponibilidad de banda ancha fija $\geq 100$ Mbps en 2018.

Fuente: Adaptado de MINECO (2019, p. 54) 
Este artículo, por lo tanto, tiene como objetivo proporcionar una visión de la situación y las posibles soluciones para el sistema educativo gallego en relación con el uso de TIC/TAC tanto dentro como fuera del aula considerando el testimonio directo del profesorado y su experiencia cuando su uso se convirtió, básicamente, en obligatorio debido a las circunstancias impuestas durante el estado de alarma.

\section{La brecha digital en Galicia}

Sparks (2013, p. 28) considera que el término brecha digital engloba una amplia gama de diferencias sociales en el acceso y uso de equipos digitales. Dewan y Riggins (2005) diseñaron un marco conceptual para ilustrar la difusión y, por lo tanto, la brecha digital de un área determinada, que establece un ciclo de adopción de las TIC basado en tres pasos: innovación en las TIC, acceso a las TIC y uso de las TIC. La tasa a la que un país o, en este caso, una comunidad autónoma, adopta nuevos avances en las TIC influye directamente en su disponibilidad para el ciudadano medio. Teniendo en cuenta la disponibilidad de banda ancha fija en Galicia según el último informe (fig. 1), la primera parte del marco de Dewan y Riggins podría estar bien orientada, pero el acceso no sería posible en muchos casos. El hecho es que las TIC se están moviendo más rápido que la infraestructura creada para ella, ya que esto no sólo depende de la innovación, sino también de factores económicos, orográficos e, incluso, culturales. Por un lado, la inversión realizada para resolver los problemas tanto por el gobierno de la comunidad autónoma (Xunta) como por el gobierno estatal podría considerarse más o menos eficiente. Sin embargo, lo cierto es que la complicada orografía y la población dispersa de las zonas rurales implican una implementación más lenta de la banda ancha fija y se necesita más y mejor planificación. El fracaso en este segundo punto obstruye la realización del tercer paso, a medida que las TIC se vuelven más innovadoras, más calidad en la banda ancha es necesaria. En consecuencia, el uso de las TIC en los hogares gallegos está mejorando, pero sigue presentando problemas en cuanto a la calidad del servicio que ralentiza las innovaciones y desmotiva a los usuarios. También deben tenerse en cuenta los factores económicos por parte de los usuarios, ya que la banda ancha no es nada sin hardware adecuado. Como han señalado varios de los profesores en este estudio (ver sección de resultados), la falta de disponibilidad de recursos (PC, portátil, tableta y productos similares) que tienen sus alumnos es un obstáculo para la instrucción en línea a diario y esto se ha acentuado durante el confinamiento.

\section{a. Acceso a internet y uso de las TIC/TAC}

Considerar que el hecho de tener acceso a internet implica también acceso a las TIC/TAC en igualdad de condiciones para todos es, básicamente, una falacia. La mayoría de las personas pueden utilizar sus teléfonos inteligentes como puntos de acceso a la red, pero esto no significa que sepan cómo utilizarlos con fines didácticos o informativos (Mendoza Ruano \& Caldera Serrano, 2014; Sparks, 2013; van Deursen \& van Dijk, 2009) o que puedan hacer un uso activo de ellos (Nemer, 2015). De hecho, Nemer (2015) insta a la necesidad de políticas de inclusión digital para apoyar a esa parte de la sociedad que ha sido marginada de la sociedad de la información por falta de recursos y conocimientos tecnológicos.

Otra de las principales necesidades para el correcto funcionamiento de las TIC/TAC en el aula, ya sea presencial o en línea, es el dominio del profesor de las mismas herramientas. Mathiasen y Schrum (2010, p. 393) señalaron este factor como uno de los retos del sistema educativo. Sparks (2013) confía en la educación formal como motor de cambio para ello, proporcionando soluciones a partir de su experiencia directa de la situación. La formación de los profesores es sólo un paso en la adopción de un enfoque más tecnológico de la educación, ya que los estudiantes y las familias 
también necesitan entender esta tecnología. El acceso y la comprensión de las herramientas TIC forman parte de la educación inclusiva (Cabero Almenara \& Ruíz Palmero, 2018). Es esencial proporcionar a la sociedad equidad en este campo para evitar el agravamiento de la brecha digital en la enseñanza. Dar por sentado la accesibilidad a internet es peligroso, pero, suponer que todos nuestros estudiantes pueden tener su propio ordenador es no ser conscientes de la situación de todas las familias. A pesar de que la mayoría de las personas tienen un teléfono inteligente o tableta, esto no significa que estos dispositivos deben ser considerados como adecuados para la enseñanza en línea. Si el objetivo de la educación en esta década es lograr un correcto funcionamiento y uso de los recursos TIC/TAC, el cambio debe involucrar tanto a las familias como a los profesores.

\section{b. La influencia de la edad}

La brecha digital es también un problema relacionado con la edad. A pesar de que se espera que los docentes aprendan y mejoren toda su vida laboral en aras de una mejor práctica docente, el alumnado está mucho más en contacto con las nuevas tecnologías (Tapscott, 2009; Avila Muñoz \& Santos Díaz, 2019). Si los estudiantes están en la adolescencia, la brecha digital entre profesores y estudiantes es aún más aguda, ya que éstos forman parte de la Generación Z, siendo parte de la primera generación de adolescentes considerados nativos digitales, esto es, nacidos entre 1995 y 2010. La tecnología es algo que los estudiantes esperan en el aula ya que forma parte de su vida cotidiana (Prensky, 2001, Torrado Cespón, 2015). Sin embargo, no todos sus profesores poseen el nivel necesario de competencia digital ya que son inmigrantes digitales y, por lo tanto, están influidos por el factor de la edad y, como señalan Busquet i Duran, Munté Ramos y Garrido Lora (2016) separados de sus alumnos en términos socioculturales. Un profesor que ha estado en activo desde los años 80 ha pasado por muchos cambios en el sistema educativo. Algunos de estos cambios, como el uso cada vez mayor de las TIC/TAC, resultan abrumadores para algunos de ellos si no se les proporciona la formación correcta. Por lo tanto, no se trata de una cuestión de capacidad de aprendizaje, sino de una cuestión relacionada con el hecho de que pertenecen a una generación diferente cuando las tecnologías no eran muy frecuentes en la enseñanza (Prensky, 2001; Van Deursen, 2007). Por otro lado, es importante tener en cuenta que la edad cronológica y la edad cognitiva no son paralelas. Al estar influida por las experiencias del individuo y de estilo de vida cognitivo, los profesores que trabajan para mejorar su estilo de enseñanza y sus recursos estarían previsiblemente menos afectados por la edad que otros adultos que no son cognitivamente activos (Barak \& Rahtz, 1990; Peral Peral, Arenas Gaitan \& Villarejo Ramos, 2015). Sin embargo, menos afectado significa simplemente menos y no sin importancia, por lo que el factor de edad es una variable relevante en la formación de los profesores que debe analizarse. La motivación aquí juega un papel importante en la adquisición de nuevos conocimientos, evitando algunas de las implicaciones negativas de este factor, ya que la formación continua y las ansias de adaptarse a nuevas corrientes educativas se convierten en una decisión consciente con el objetivo de mejorar una situación (Dörnyei \& Ushioda, 2011). Por lo tanto, motivar a los profesores para que aprendan la correcta aplicación de las TIC/TAC es tan importante como proporcionarles una formación adecuada. 


\section{Metodología}

Teniendo en cuenta los factores relacionados con la brecha digital en Galicia, de marzo a abril de 2020, un total de 168 institutos gallegos recibieron un cuestionario online invitando a todos sus profesores a ofrecer su visión de la presencia y uso de las TIC/TAC en su vida laboral diaria antes y durante el confinamiento. A finales de mayo de 2020, el número de respuestas válidas era de 201.

\section{a. Instrumentos}

Como método para recabar datos en la sociedad de la información, el uso de herramientas TIC en línea es un recurso evidente. Los cuestionarios de Google ofrecían la posibilidad de obtener resultados claros y su distribución es rápida y fácil. Las preguntas incluidas dirigidas a conocer la situación real de los profesores de secundaria tienen en cuenta varios factores y teniendo en cuenta la situación derivada del cierre de escuelas durante el estado de alarma en España:

- Años de experiencia docente.

- Formación en TIC/TAC durante sus años de grado o máster.

- Búsqueda de formación TIC/TAC después del grado o máster.

- Consideración del uso de TIC/TAC tanto dentro como fuera del aula.

- Consideración de la necesidad de cursos de formación TIC/TAC.

Se incluyó un espacio de reflexión opcional final para proporcionar más información sobre sus percepciones sobre el uso de las TIC/TAC, proporcionando un análisis cualitativo complementario de la situación.

Para el análisis de los resultados cuantitativos, todas las variables se codificaron en un documento .sav y posteriormente se analizaron utilizando SPSS. El análisis cualitativo explora las respuestas individualmente y las agrupa de acuerdo con las ideas expresadas.

\section{Resultados y discusión}

Las respuestas al cuestionario se han analizado teniendo en cuenta las relaciones entre las variables descritas. Para proporcionar una mejor comprensión de la situación, los resultados aparecen en tres bloques principales que engloban las preguntas del cuestionario: uso de las TIC/TAC, la influencia de la edad y la formación. Más tarde se complementan con el análisis cualitativo de las opiniones de los profesores.

\section{a. Uso de las TIC/TAC antes y durante el confinamiento}

En primer lugar, en números absolutos, el uso de las TIC/TAC por parte de los profesores antes y durante el confinamiento es significativamente alto (tabla 1) pero no en la misma medida. La tabla 1 muestra cómo el porcentaje de uso de las TIC en el hogar aumentó considerablemente durante el confinamiento, sin embargo, esto muestra una percepción errónea de la realidad, ya que la situación obligó a los docentes a usar la tecnología incluso cuando no estaban listos, o se sentían abrumados como muestran sus testimonios (véase d.b.). Por lo tanto, el uso extensivo de las TIC/TAC durante este período no significa que los problemas percibidos anteriormente por ese $48,26 \%$ de los profesores (suma de los que utilizaron las TIC/TAC nunca o casi nunca o rara vez para tareas fuera del aula, tabla 1) hubiesen desaparecido: sólo significa que era la única manera en que po- 
dían contactar a sus alumnos durante ese período. En algunos casos, esto implicaba una formación acelerada y forzada que no era satisfactoria ni para los estudiantes ni para los profesores, especialmente cuando la razón principal para evitar el uso de las TIC/TAC para tareas domésticas (tabla 2 ) es que sus alumnos carecen de recursos, lo que es un claro reflejo de lo que la brecha digital implica para las familias. Después de revisar los comentarios, varios docentes también expresaron esta preocupación (véase d.c.). Por lo tanto, esto muestra cómo el uso de las TIC/TAC durante el confinamiento se convirtió en una especie de imposición, siendo algunos profesores seguros de que sus alumnos no podían acceder a los recursos en igualdad de condiciones. En algunas ciudades, el gobierno local proporcionó a los estudiantes ordenadores y recursos de impresión para minimizar la brecha digital, pero en realidad era una manera de enfatizar la necesidad de una mayor intervención e inversión en la calidad y el acceso a las TIC por parte de la población. También es importante tener en cuenta las necesidades de los diferentes sujetos y cómo se vieron afectados por el confinamiento (tabla 3). Si tanto los estudiantes como los profesores estaban acostumbrados a emplear las TIC/TAC como parte estándar de su rutina de enseñanza, el cambio no fue significativo. Por otra parte, los sujetos que se basaban en una instrucción cara a cara más directa se vieron altamente afectados por el cambio. Así, mientras que algunas asignaturas presentan algunas diferencias en el uso de las TIC/TAC en el aula y para las tareas domésticas (tabla 3), la mayoría de los profesores recurrió a ellas durante el confinamiento (tabla 1). Por lo tanto, a pesar de que el porcentaje de uso frecuente (cada semana o todos los días) es considerablemente alto en la mayoría de las asignaturas, los docentes parecen enfatizar su uso en el aula en lugar de en casa.

\begin{tabular}{|l|c|c|c|c|}
\hline \multirow{2}{*}{ Uso } & \multicolumn{3}{|c|}{ Frecuencia y porcentaje } \\
\cline { 2 - 5 } & $\begin{array}{c}\text { Nunca o casi } \\
\text { nunca }\end{array}$ & $\begin{array}{c}\text { Una o dos veces } \\
\text { al mes }\end{array}$ & $\begin{array}{c}\text { Todas las se- } \\
\text { manas }\end{array}$ & Todos los días \\
\hline En el aula & $20(9.95 \%)$ & $34(16.92 \%)$ & $58(28.86 \%)$ & $89(44.28 \%)$ \\
\hline Para tareas fuera del aula & $61(30.35 \%)$ & $36(17.91 \%)$ & $53(26.37 \%)$ & $51(25.37 \%)$ \\
\hline Durante el confinamiento & \multicolumn{2}{|c|}{$7(3.48 \%)$} & $194(96.52 \%$ \\
\hline
\end{tabular}

Tabla 1. Uso de las TIC/TAC.

\begin{tabular}{|l|c|}
\hline & Frecuencia y porcentaje \\
\hline Falta de conocimientos & $11(18.03 \%)$ \\
\hline Falta de recursos & $2(3.27 \%)$ \\
\hline Mis alumnos no tienen recursos & $36(59.01 \%)$ \\
\hline No quiero/no es necesario & $12(19.67 \%)$ \\
\hline
\end{tabular}

Tabla 2. Razones para no usar TIC/TAC para tareas fuera del aula. 


\begin{tabular}{|c|c|c|c|c|c|c|}
\hline \multirow[t]{2}{*}{ Asignatura } & \multirow[t]{2}{*}{ No } & \multirow[t]{2}{*}{ Uso } & \multicolumn{4}{|c|}{ Frecuencia y porcentaje } \\
\hline & & & $\begin{array}{c}\text { Nunca o casi } \\
\text { nunca }\end{array}$ & $\begin{array}{c}\text { Una o dos } \\
\text { veces al mes }\end{array}$ & $\begin{array}{l}\text { Todas las } \\
\text { semanas }\end{array}$ & Todos los días \\
\hline \multirow[t]{2}{*}{ Lengua gallega } & \multirow[t]{2}{*}{19} & En el aula & $1(5,3 \%)$ & $4(21,1 \%)$ & $6(31,6 \%)$ & $8(42,1 \%)$ \\
\hline & & $\begin{array}{l}\text { Fuera del } \\
\text { aula }\end{array}$ & $5(26,3 \%)$ & $6(31,6 \%)$ & $7(36,8 \%)$ & $1(5,3 \%)$ \\
\hline \multirow[t]{2}{*}{ Lengua española } & \multirow[t]{2}{*}{20} & En el aula & $4(20 \%)$ & $5(25 \%)$ & $6(30 \%)$ & $5(25)$ \\
\hline & & $\begin{array}{l}\text { Fuera del } \\
\text { aula }\end{array}$ & $9(45 \%)$ & $3(15 \%)$ & $5(25 \%)$ & $3(15 \%)$ \\
\hline \multirow[t]{2}{*}{ Lengua inglesa } & \multirow[t]{2}{*}{25} & En el aula & $1(4 \%)$ & $6(24 \%)$ & $4(16 \%)$ & $14(56 \%)$ \\
\hline & & $\begin{array}{l}\text { Fuera del } \\
\text { aula }\end{array}$ & $1(5,3 \%)$ & $4(21,1 \%)$ & $6(31,6 \%)$ & $8(42,1 \%)$ \\
\hline \multirow[t]{2}{*}{ Lengua alemana } & \multirow[t]{2}{*}{2} & En el aula & $0(0 \%)$ & $0(0 \%)$ & $2(100 \%)$ & $0(0 \%)$ \\
\hline & & $\begin{array}{l}\text { Fuera del } \\
\text { aula }\end{array}$ & $0(0 \%)$ & $1(50 \%)$ & $1(50 \%)$ & $0(0 \%)$ \\
\hline \multirow[t]{2}{*}{ Lengua francesa } & \multirow[t]{2}{*}{9} & En el aula & $4(44,4 \%)$ & $0(0 \%)$ & $2(22,2 \%)$ & $3(33,3 \%)$ \\
\hline & & $\begin{array}{l}\text { Fuera del } \\
\text { aula }\end{array}$ & $6(66,7 \%)$ & $3(33,3 \%)$ & $0(0 \%)$ & $0(0 \%)$ \\
\hline \multirow[t]{2}{*}{ Latín / Griego } & \multirow[t]{2}{*}{5} & En el aula & $1(20 \%)$ & $1(20 \%)$ & $1(20 \%)$ & $2(40 \%)$ \\
\hline & & $\begin{array}{l}\text { Fuera del } \\
\text { aula }\end{array}$ & $1(20 \%)$ & $0(0 \%)$ & $2(40 \%)$ & $2(40 \%)$ \\
\hline \multirow[t]{2}{*}{ Ciencias } & \multirow[t]{2}{*}{1} & En el aula & $0(0 \%)$ & $0(0 \%)$ & $1(100 \%)$ & $0(0 \%)$ \\
\hline & & $\begin{array}{l}\text { Fuera del } \\
\text { aula }\end{array}$ & $1(100 \%)$ & $0(0 \%)$ & $0(0 \%)$ & $0(0 \%)$ \\
\hline \multirow[t]{2}{*}{ Matemáticas } & \multirow[t]{2}{*}{23} & En el aula & $3(13 \%)$ & $5(21,7 \%)$ & $8(34,8 \%)$ & $7(30,4 \%)$ \\
\hline & & $\begin{array}{l}\text { Fuera del } \\
\text { aula }\end{array}$ & $0(0 \%)$ & $8(34,8 \%)$ & $5(21,7 \%)$ & $10(43,5 \%)$ \\
\hline \multirow[t]{2}{*}{ Tecnología } & \multirow[t]{2}{*}{16} & En el aula & $0(0 \%)$ & $0(0 \%)$ & $3(18,8 \%)$ & $13(81,3 \%)$ \\
\hline & & $\begin{array}{l}\text { Fuera del } \\
\text { aula }\end{array}$ & $2(12,5 \%)$ & $3(18,8 \%)$ & $6(37,5 \%)$ & $5(31,3 \%)$ \\
\hline \multirow{2}{*}{$\begin{array}{l}\text { Historia / geogra- } \\
\text { fía }\end{array}$} & \multirow[t]{2}{*}{17} & En el aula & $1(5,9 \%)$ & $2(11,8 \%)$ & $5(29,4 \%)$ & $9(52,9 \%)$ \\
\hline & & $\begin{array}{l}\text { Fuera del } \\
\text { aula }\end{array}$ & $2(11,8 \%)$ & $2(11,8 \%)$ & $6(35,3 \%)$ & $7(41,2 \%)$ \\
\hline \multirow[t]{2}{*}{ Física / química } & \multirow[t]{2}{*}{5} & En el aula & $0(0 \%)$ & $0(0 \%)$ & $2(40 \%)$ & $3(60 \%)$ \\
\hline & & $\begin{array}{l}\text { Fuera del } \\
\text { aula }\end{array}$ & $0(0 \%)$ & $2(40 \%)$ & $1(20 \%)$ & $2(40 \%)$ \\
\hline Biología & 2 & En el aula & $1(4,5 \%)$ & $0(0 \%)$ & $10(45,5 \%)$ & $11(50 \%)$ \\
\hline
\end{tabular}




\begin{tabular}{|c|c|c|c|c|c|c|}
\hline & & $\begin{array}{c}\text { Fuera del } \\
\text { aula }\end{array}$ & $7(31,8 \%)$ & $7(31,8 \%)$ & $5(22,7 \%)$ & $3(13,6 \%)$ \\
\hline \multirow[t]{2}{*}{ Filosofía } & \multirow[t]{2}{*}{4} & En el aula & $0(0 \%)$ & $2(50 \%)$ & $1(25 \%)$ & $1(25 \%)$ \\
\hline & & $\begin{array}{c}\text { Fuera del } \\
\text { aula }\end{array}$ & $2(50 \%)$ & $0(0 \%)$ & $1(25 \%)$ & $1(25 \%)$ \\
\hline \multirow[t]{2}{*}{ Educación física } & \multirow[t]{2}{*}{11} & En el aula & $1(9,1 \%)$ & $6(54,5 \%)$ & $3(27,3 \%)$ & $1(9,1 \%)$ \\
\hline & & $\begin{array}{c}\text { Fuera del } \\
\text { aula }\end{array}$ & $5(45,5 \%)$ & $2(18,2 \%)$ & $3(27,3 \%)$ & $1(9,1 \%)$ \\
\hline \multirow[t]{2}{*}{ Economía } & \multirow[t]{2}{*}{6} & En el aula & $1(16,7 \%)$ & $0(0 \%)$ & $2(33,3 \%)$ & $3(50 \%)$ \\
\hline & & $\begin{array}{c}\text { Fuera del } \\
\text { aula }\end{array}$ & $2(33,3 \%)$ & $0(0 \%)$ & $2(33,3 \%)$ & $2(33,3 \%)$ \\
\hline \multirow[t]{2}{*}{ Educación plástica } & \multirow[t]{2}{*}{9} & En el aula & $3(33,3 \%)$ & $1(11,1 \%)$ & $1(11,1 \%)$ & $4(44,4 \%)$ \\
\hline & & $\begin{array}{c}\text { Fuera del } \\
\text { aula }\end{array}$ & $3(33,3 \%)$ & $1(11,1 \%)$ & $1(11,1 \%)$ & $4(44,4 \%)$ \\
\hline \multirow[t]{2}{*}{ Música } & \multirow[t]{2}{*}{3} & En el aula & $1(33,3 \%)$ & $0(0 \%)$ & $0(0 \%)$ & $2(66,7 \%)$ \\
\hline & & $\begin{array}{c}\text { Fuera del } \\
\text { aula }\end{array}$ & $1(33,3 \%)$ & $0(0 \%)$ & $0(0 \%)$ & $2(66,7 \%)$ \\
\hline \multirow[t]{2}{*}{ Religión } & \multirow[t]{2}{*}{4} & En el aula & $0(0 \%)$ & $2(50 \%)$ & $1(25 \%)$ & $1(25 \%)$ \\
\hline & & $\begin{array}{l}\text { Fuera del } \\
\text { aula }\end{array}$ & $0(0 \%)$ & $2(50 \%)$ & $2(50 \%)$ & $0(0 \%)$ \\
\hline
\end{tabular}

Tabla 3. Uso de las TIC/TAC por asignatura

\section{b. La influencia de la edad en el uso de las TIC/TAC}

Las actitudes y el uso de las TIC/TAC demostradas en esta investigación por parte de los profesores que habían estado en activo durante más tiempo pueden dar una idea aproximada de la forma en que se enfrentan a la necesidad de reinventar su estilo de enseñanza. La existencia del factor de edad también es percibida por los propios docentes. Por ejemplo, uno de ellos señala:

SEC133 Entre la generación "no digital" como la mía, una gran parte del profesorado todavía no sabe cómo poner sus páginas favoritas del navegador en la barra de marcadores. Ante un mensaje de error o una pregunta donde hay dos opciones para responder, se quedan atascados. Introducirlos en algo nuevo es muy difícil porque no tienen la mínima "intuición o curiosidad digital". Me consta que la mayoría están haciendo un esfuerzo extra en estos días de confinamiento para que los alumnos no se queden desconectados. Se requiere muchísima paciencia para ayudarlos con las herramientas más básicas. Pienso que entre los profesores más jóvenes, hay de todo. El ingrediente más importante para usar herramientas digitales es la paciencia y conocer el sistema operativo que usan. ¿Cómo se pueden utilizar algunos de esos recursos, como Vocaroo (en nuestro caso, con Webex), cuando el micrófono no nos funciona y no sabemos dónde buscar en la configuración de Windows?

Un total de 86 profesores que habían estado en activo durante más de 25 años respondieron al cuestionario. Sin embargo, han resultado ser usuarios considerablemente activos de las TIC/TAC tanto dentro como fuera del aula (tabla 4). La tabla 4 también muestra cómo la diferencia de uso 
dentro y fuera del aula no es significativa estadísticamente, pero en realidad lo es en términos sociales. La razón principal indicada por los profesores para la no utilización de las TIC/TAC para tareas domésticas es que sus alumnos carecen de recursos, o carecen de conocimiento, como se explicó anteriormente (tabla 2).

En cuanto a la formación en TIC/TAC, los profesores de este bloque demostraron haber superado los problemas asociados con que el factor de edad estando cognitivamente activos. Como se señala en la revisión de la literatura, la motivación es esencial en este caso. La experiencia de estos profesores es muy valiosa para las generaciones futuras de profesores, demostrando, una vez más, cómo se trata de un trabajo vocacional en el que, sin importar los obstáculos, los profesores comprometidos siempre intentarán superarlos. Como algunos de estos profesores experimentados manifestaron:

SEC12: La necesidad obliga. Lo he constatado con esta dramática situación. La conclusión es clara: si quieres, puedes. Las TIC no se comen a nadie, al contrario (en activo desde 1988).

SEC100: como siempre, el docente medio debe tirar de experiencia o autoaprendizaje por la falta de previsión de las autoridades docentes competentes. No es nada nuevo, pero si constante (en activo desde 1990).

Obviamente, estos docentes han pasado por muchos cambios en su vida laboral, por lo que su experiencia también es un factor a tener en cuenta a la hora de decidir qué es mejor para sus asignaturas y grupos de acuerdo a sus percepciones. Así, los resultados (tabla 4) muestran cómo el factor de edad no es relevante con respecto al uso de TIC/TAC en el aula. Además, la mayoría de ellos han buscado recursos para mejorar su competencia digital (83 de 86) En consecuencia, los datos apoyan los hallazgos de Barak y Rahtz (1990), y Peral Peral, Arenas Gaitan y Villarejo Ramos (2015).

\begin{tabular}{|l|l|c|c|c|c|}
\hline \multirow{2}{*}{ Periodo } & \multirow{2}{*}{ No } & \multicolumn{2}{|c|}{ Uso en el aula } & \multicolumn{2}{|c|}{ Uso para tareas fuera del aula } \\
\cline { 3 - 6 } & & $\begin{array}{c}\text { Diaria o sema- } \\
\text { nalmente }\end{array}$ & $\begin{array}{c}\text { Rara vez o } \\
\text { nunca }\end{array}$ & $\begin{array}{c}\text { Diaria o sema- } \\
\text { nalmente }\end{array}$ & $\begin{array}{c}\text { Rara vez o } \\
\text { nunca }\end{array}$ \\
\hline $1980-1984$ & 18 & $100 \%$ & $0 \%$ & $71.43 \%$ & $28.57 \%$ \\
\hline $1985-1989$ & 36 & $63.89 \%$ & $36.11 \%$ & $61.11 \%$ & $38.89 \%$ \\
\hline $1990-1994$ & 32 & $74.07 \%$ & $25.93 \%$ & $55.56 \%$ & $44.44 \%$ \\
\hline
\end{tabular}

Tabla 4. Profesores en activo desde hace más de 25 años.

\section{c. Formación en TIC/TAC}

La formación en TIC/TAC es decisiva para un uso adecuado de los recursos en la enseñanza. Como se espera, la mayoría de los profesores han buscado algún tipo de formación, respondiendo de forma afirmativa 192 docentes participantes (95,52 \%). Es interesante observar que la forma más típica de buscar esta formación ha sido llevar a cabo su propia exploración autodidacta de las TIC/ TAC (tabla 5). 


\begin{tabular}{|c|c|c|c|c|c|c|c|c|c|}
\hline \multicolumn{2}{|c|}{ Autodidacta } & \multicolumn{2}{|c|}{$\begin{array}{l}\text { Cursos presenciales } \\
\text { de entidades edu- } \\
\text { cativas }\end{array}$} & \multicolumn{2}{|c|}{$\begin{array}{c}\text { Cursos online de } \\
\text { entidades educati- } \\
\text { vas }\end{array}$} & \multicolumn{2}{|c|}{$\begin{array}{c}\text { Cursos presenciales } \\
\text { de la Consellería o } \\
\text { Ministerio }\end{array}$} & \multicolumn{2}{|c|}{$\begin{array}{c}\text { Cursos online de la } \\
\text { Consellería o Minis- } \\
\text { terio }\end{array}$} \\
\hline Sí & No & Sí & No & Sí & No & Sí & No & Sí & No \\
\hline $\begin{array}{l}152 \\
(79,2 \%\end{array}$ & $\begin{array}{l}40 \\
(20,8 \%\end{array}$ & $\begin{array}{l}84 \\
(43,8 \%\end{array}$ & $\begin{array}{l}108 \\
(56,3 \%\end{array}$ & $\begin{array}{c}83 \\
(43,2 \%\end{array}$ & $\begin{array}{c}109 \\
(56,8 \%\end{array}$ & $\begin{array}{c}106 \\
(55,2 \%\end{array}$ & $\begin{array}{c}86 \\
(44,8 \%\end{array}$ & $\begin{array}{c}90 \\
(46,9 \%\end{array}$ & $\begin{array}{c}102 \\
(53,1 \%\end{array}$ \\
\hline ) & ) & ) & ) & ) & ) & ) & ) & ） & ） \\
\hline
\end{tabular}

Tabla 5. Formación de los participantes.

La percepción de la necesidad de una mayor formación entre los participantes también está clara, ya que 197 (98\%) manifestó tal necesidad. Entre las posibilidades que se ofrecen en el cuestionario, la formación opcional es la que tiene el mayor número de simpatizantes (tabla 6). Además, la mayoría manifiesta la necesidad de complementarla ya sea durante la realización de sus estudios o incluso mediante el establecimiento de cursos obligatorios.

\begin{tabular}{|l|l|l|}
\hline Durante el grado o postgrado & Cursos obligatorios & Cursos opcionales \\
\hline $90(44,8 \%)$ & $92(45,8 \%)$ & $120(59,7 \%)$ \\
\hline
\end{tabular}

Tabla 6. Formación del profesorado.

\section{d. Análisis cualitativo}

La última parte del cuestionario incluía espacio libre donde los participantes podían añadir información adicional para complementar sus respuestas y proporcionar más énfasis en algunas esferas. Un total de $66(36,8 \%)$ sujetos hizo uso de esta sección y sus preocupaciones se dividen en cuatro bloques principales (nótese que la respuesta de algunos sujetos puede identificarse con varios bloques):

- Falta de recursos $(42,4 \%)$

- Falta de formación $(33,3 \%)$

- Falta de recursos en los hogares de los estudiantes $(13,6 \%)$

- Falta de tiempo $(7,5 \%)$

- Factor afectivo $(4.5 \%$

\section{Falta de recursos}

En cuanto a los recursos, la mayoría de los profesores reflexionan críticamente sobre el hecho de que se les pide que utilicen Las TIC/TAC, pero las instituciones no les han proporcionado material (SEC74, SEC 118) ni Internet está disponible en todos los hogares (SEC188, SEC196):

- SEC74: Sobre todo, hace falta más medios y más eficaces.

- SEC118: El obstáculo principal no es la formación sino los medios a disposición. Hay grupos con los que NUNCA tengo acceso a la sala de PC. Y con los demás, solo una vez por semana con un número de PC insuficientes. Así no hay ni motivación ni motivo para mayor formación.

- SEC188: La mala cobertura de internet en las zonas rurales imposibilita hacer uso de las TIC/TAC. 
- SEC196: Es necesario, que la Xunta nos garantice a los docentes que en nuestro domicilio tengamos una conexión a internet de calidad.

\section{Falta de formación}

Varios participantes comentan la necesidad de una mayor formación, lo que proporciona una imagen clara de cómo se sienten acerca de su competencia. Entre ellas, cabe destacar la demanda de cuatro acciones diferentes: actualización de recursos, disponibilidad, viabilidad y asesoramiento. Estas necesidades que se pueden resumir en las siguientes opiniones:

- SEC19: Todos los centros deberían tener un Aula Virtual con un formato actualizado (la que hay de la Xunta es de hace diez años, está obsoleta y no es fácil de utilizar ni por profes ni por niños). Los cursos que hay online en FProfe para la formación de los profesores son un chiste para hacer puntos y no para formar al profesorado, que es lo que necesitamos. No necesitamos una presión asfixiante para formarnos en las TIC, acudiendo a cursos fuera del horario laboral obligatoriamente, pero sí que exista esa posibilidad con plazas para todos, pues el aforo de muchos cursos es muy limitado y nunca hay plazas para todos los que estamos interesados. Además, es injusto que haya cursos distintos de unas ciudades a otras.

- SEC34: La formación del máster en profesorado es muy teórica y poco práctica, debería mejorarse este aspecto.

- SEC121: Necesitamos una hora semanal con un profesional para que nos explique paso a paso el funcionamiento de distintas aplicaciones, o por lo menos tener a nuestra disposición a alguien a quien preguntarle dudas y nos mantenga actualizado.

\section{Falta de recursos en los hogares de los estudiantes}

Por otro lado, los profesores manifiestan sus preocupaciones sobre los recursos que sus alumnos tienen en casa, como ya se ha señalado anteriormente (tabla 2), que implican que, hagan el esfuerzo que hagan, si no pueden llegar a sus alumnos, no vale de nada:

- SEC16: Hay muchos alumnos que no siguen el aula virtual ni la página web del centro porque no tienen wifi y los padres necesitan el ordenador para trabajar.

- SEC33: Vergonzosas las dificultades del alumnado para acceder a la red y disponer de dispositivos que les permitan trabajar desde casa.

- SEC147: Si las familias no tienen ingresos, las nuevas tecnologías son un gasto añadido. No es básico como la alimentación y los productos de limpieza.

\section{Falta de tiempo}

Los docentes también señalan la falta de tiempo. Ante un sistema que incluye ratios de 30 alumnos (más repetidores) por aula, esta situación impide considerar las diferentes necesidades de los alumnos. En consecuencia, al pedir a los profesores que dediquen más tiempo a acciones formativas implica un grado de saturación que afectará negativamente a su práctica docente:

- SEC36: La razón más poderosa para no usar con mayor frecuencia las TIC, en mi caso, es la sobrecarga de horas de docencia y la ratio de alumnado, que complica la exploración de opciones por mi cuenta y la implementación de nuevas actividades, pues a duras penas consigo llegar a todo y a todos. También tiene peso el perfil de mi alumnado, pues su digitaliza- 
ción es menos "real" de lo que parece, y fundamentalmente escorada al ámbito de los juegos.

- SEC119: La extensión de los currículos hace que el tiempo sea muy limitado para el uso diario de las herramientas TIC en el aula.

- SEC189: La carga de trabajo ADMINISTRATIVO es cada vez mayor y la carga lectiva no deja de subir. Esto dificulta muchísimo la formación continua del profesorado.

\section{Factor afectivo}

Además de estas reflexiones sobre el uso (o mal uso) de las TIC/TAC en la práctica docente, también vale la pena considerar el papel del factor afectivo y el apego. Tres profesores expresan su preocupación por el aprendizaje basado en las TIC/TAC:

- SEC51: La docencia telemática nunca podrá substituir a la docencia presencial por tantas razones que en este espacio no puedo ni empezar a dilucidar, pero el motivo principal es el aprendizaje de convivir y de tolerar a nuestros iguales.

- SEC71: Está muy bien conocer las herramientas de las tecnologías de la información y comunicación. Es importante tener una formación en esto y para solventar situaciones como la que vivimos vienen fenomenal. Pero deben ser un apoyo, un complemento, no creo que haya que basar toda la educación en las TAC/TIC.

- SED75: Un ordenador no substituye al profesorado.

Estos testimonios son especialmente relevantes pese a su bajo número al conectar con la idea de alienación entre el profesorado y el alumnado en ambientes online (Dickey, 2004; Rasheed, Kamsin \& Abdullah, 2020; Tichavsky et al. 2015) y apoyar la existencia y la necesidad del lado afectivo en el proceso de aprendizaje). Los profesores son percibidos como modelos de comportamiento para sus estudiantes, no necesariamente como modelos que quieran seguir, sino alguien que está jerárquicamente por encima de ellos. Los profesores deben ser considerados como la autoridad en el aula, a pesar de que los estudiantes tengan un comportamiento disruptivo, o que el docente no pueda, simplemente, manejarlos. La enseñanza en línea evita todos estos problemas. Sin embargo, esto no es positivo. Eliminar la posibilidad de interacción social en el aula también evita la aparición de soluciones para cualquier tipo de posible problema social derivado de esta interacción que podría aplicarse fuera del aula. Las escuelas (institutos, en este caso) no son sólo centros para el aprendizaje de contenido académico, sino también habilidades sociales y respeto además de un lugar adecuado para trabajar en la mediación y la incomprensión intercultural (Scollon \& WongScollon, 2001; Beacco et al., 2016; Consejo de Europa, 2018). Como docentes, somos responsables de ayudar a las mentes del futuro a dar sus primeros pasos en el mundo del conocimiento lejos de los dogmatismos, cuestionando y respondiendo de una manera constructiva y esto necesita contacto. Por lo tanto, si la adquisición de habilidades de pensamiento crítico ayuda a los estudiantes a dar forma a cómo interactúan entre sí en el entorno de aprendizaje (Nicolini, 2008) y cómo, más adelante, pueden aplicar esta experiencia a situaciones fuera del aula, la enseñanza en línea les priva de esta formación social. Sin embargo, la Generación Z podría estar cambiando esta percepción de las relaciones sociales. Como Turner (2015, p. 109) señaló, la Generación Z utiliza las tecnologías como una forma de alejarse de situaciones sociales incómodas y esto podría inhibir sus habilidades sociales en la interacción cara a cara. Ya sea positivo o no, tal vez este sea un cambio que la esa parte de la sociedad no digital debe admitir. 


\section{Conclusiones}

La falta de preparación demostrada por los docentes apoya la necesidad de una mayor inversión en el aprendizaje de larga duración y múltiples oportunidades para actualizar sus habilidades. Esta situación ya fue señalada por Mathiasen y Schrum (2010) una década antes, pero aún no se ha resuelto. La brecha digital, tanto en los hogares de profesores como en los hogares de los estudiantes, no permite un rápido avance en la instauración de las TIC/TAC como una posibilidad universal de enseñanza. La situación vivida debido al confinamiento ha hecho la situación más evidente y el sueño de una enseñanza online en igualdad de condiciones para todos basado en herramientas en línea sigue siendo utópico. Aunque según Sparks (2013, p. 39) ya adelantaba que la desaparición de la brecha digital está lejos de ser posible, puesto que no sólo depende de la infraestructura, sino también de la sociedad, lo cierto es que conocer las necesidades de los docentes puede ayudar a las instituciones en sus primeros pasos para buscar soluciones. Si tratamos de acelerar el proceso, los resultados pueden ser desastrosos, destruyendo la motivación y, en general, derivando en el mal uso de los recursos y el tiempo.

Teniendo en cuenta la necesidad actual de formación sobre TIC/TAC, también es necesario pensar en los recursos en línea y las metodologías de enseñanza que pueden satisfacer las necesidades de los estudiantes (Area, 2009; Area \& Adell, 2009; Pallof \& Pratt, 2003; Calderero Hernández, Aguirre Ocaña, Castellanos Sánchez, Peris Sirvent \& Perochena González, 2014; Cabero \& Barroso, 2016; Escudero; Martínez-Domínguez \& Nieto, 2018). Esto significa que las TIC/TAC no sólo necesitas estar disponible, sino también ser las adecuadas. Desde rutas de aprendizaje online (Castillo Rodríguez \& Torrado Cespón, 2020) que se pueden modelar de acuerdo no sólo al grupo, sino también a estudiantes individuales, hasta la existencia misma de sitios especializados (considérese la revisión de Castillo Rodríguez y Arias Montañana (2018) como ejemplo para la enseñanza del inglés) a webs o apps asociadas a todo un tema, como el EPSS Multimedia Lab, formado de acuerdo con las necesidades fonológicas de los estudiantes universitarios españoles de inglés y que se puede utilizar de forma independiente (Gómez González, Sánchez Roura, Torrado Cespón, Rollings \& Gómez Penas, 2016). Sin embargo, vale la pena repensar el proceso y reflexionar sobre lo que estamos tratando de hacer. Si el uso de las TIC/TAC sólo está dando a la enseñanza una versión digital de las prácticas anteriores, tal vez la innovación y el reconocimiento de la diversidad que estamos anhelando no es tal. Gardner y Davies (2012, cap. 7) incluso establecieron ciertos paralelismos entre esta situación y las teorías de comportamiento de Skinner, mediante la cual se perpetúan las metodologías antiguas, pero en un entorno diferente. También vale la pena considerar que la primera generación de TIC/TAC reales serán llevadas a cabo por aquellos que son nativos digitales y así superar el problema de aquellos que una vez estuvieron al otro lado de la tecnología.

La enseñanza en línea promueve el uso de un aprendizaje más autónomo por parte de los alumnos y metodologías como el aula invertida son relevantes en este caso (Tourón \& Santiago, 2015; Romero-García, Buzón-García \& Tourón, 2018). Así, la aplicación de las TIC/TAC tanto como dentro como fuera del aula no sólo está relacionada con los recursos, que son una parte esencial de la enseñanza, sino también con la competencia digital del profesorado. Es necesario analizar esto para ofrecer una mejor percepción de la situación. Para ello, cabe considerar el cuestionario diseñado y validado por Tourón, Martín, Navarro, Pradas y Iñigo (2018) para seguir investigando. Una imagen clara de la situación de los profesores ayudará a proporcionar la formación correcta no sólo para resolver situaciones similares, sino también para considerar el uso de las TIC/TAC como parte natural del proceso de enseñanza en la educación actual.

Por otro lado, si la instrucción cara a cara facilita la introducción de un aprendizaje significativo confrontado con la enseñanza en línea sigue siendo una pregunta difícil. Considerando el aula como un entorno no auténtico donde los contenidos se exponen debido a un currículo y no a las necesidades reales de los estudiantes, un aumento en el uso de las TIC/TAC en el aula podría colaborar en este sentido a la alienación de la educación con respecto al mundo real. Los profesores, por lo 
tanto, nunca deben olvidar la importancia de la educación personalizada (Bernardo Carrasco, Javaloyes Soto \& Calderero Hernández, 2016). De esta forma, en una sociedad en la que el uso de las TIC forma parte de la vida cotidiana, promover el uso significativo de las TIC/TAC supondría una reducción de la artificialidad del proceso de aprendizaje institucionalizado. Sin embargo, al tener todavía nativos e inmigrantes digitales, la adaptación seguirá siendo parte del futuro del aprendizaje, ya que ambas partes tendrían que entenderse entre sí para llegar a la mejor solución para todos.

Cambiar el uso de las TIC/TAC tanto dentro como fuera del aula no es una decisión que una pandemia o un gobierno pueda imponer. Para tener éxito, se deben considerar tanto las necesidades de los docentes como de las familias. Ofrecer hardware y software a las escuelas no soluciona nada si los profesores no pueden dedicar tiempo a la formación en nuevas tecnologías, y los estudiantes no pueden manejar los recursos TIC/TAC si no pueden acceder a equipos y banda ancha. Es necesario más inversión en educación, pero no sólo en recursos materiales, sino también en recursos humanos.

\section{Reconocimientos}

VILEO (RETOS de investigación, Universidad Internacional de La Rioja, 2020-2022) y e-LPHON4L, (RETOS 2019, Programa Estatal de I+D+i, Coofinanciamiento FEDER, 2020-2023).

\section{Referencias}

Area, M. (2009). La formación y el aprendizaje en entornos virtuales. Potencialidades, debilidades y tendencias. Crítica, 82, 33-36. Recuperado de: https://cutt.ly/Bsdz6IP

Area, M., \& Adell, J. (2009). E-learning: Enseñar y aprender en espacios virtuales. En J. De Pablos (Coord.), Tecnología Educativa. La formación del profesorado en la era de Internet (391-424). Málaga: Aljibe. Recuperado de: https://cutt.ly/3sdxS4e

Ávila Muñoz, A. M. \& Santos Díaz, I. C. (2019) Observación de la brecha tecnológica generacional desde el prisma de la disponibilidad léxica. Ogigia, revista electrónica de estudiod hispánicos, 25, 259-292. DOI: https://doi.org/10.24197/ogigia.25.2019.259-292

Barak, B., \& Rahtz, D. R. (1990). Cognitive age: Demographic and psychographic dimensions. Journal of Ambulatory care marketing, 3 (2), 51-65. DOI: http://dx.doi.org/10.1300/J273v03n02_0

Beacco, J.-C., Byram, M., Cavalli, M., Coste, D., Cuenat, M. E., Gouiller, F. \& Panthier, J. (2016). Guide for the development and implementation of curricula for plurilingual and intercultural education. Strasbourg: Council of Europe. Recuperado de: https://cutt.ly/gsdchys

Bernardo Carrasco, J.; Javaloyes Soto, J. J. \& Calderero Hernández, J. F. (2016). Cómo personalizar la educación: una solución de futuro. Narcea Ediciones. Recuperado de: https://cutt.ly/ OssDCIK

Busquet i Duran, J.; Munté Ramos, R. A. \& Garrido Lora, M. (2016) De las TIC a las TRIC. Estudio sobre el uso de las TIC y la brecha digital entre adultos y adolescentes en España. Anàlisi. Quaderns de Comunicació i Cultura, 54, 44-57. DOI: http://dx.doi.org/10.7238/a.v0i54.2953

Cabero, J., \& Ruiz-Palmero, J. (2018). Las Tecnologías de la información y la comunicación para la inclusión: reformulando la brecha digital. International Journal of Educational Research and Innovation, 9, 16-30. Recuperado de: https://www.upo.es/revistas/index.php/IJERI/article/ view/2665 
Cabero, J. \& Barroso, J. (2016) ICT Teacher Training: a View of the TPACK Model / Formación del profesorado en TIC: una visión del modelo TPACK. Culture and Education, 28 (3), 633-663. DOI: https://doi.org/10.1080/11356405.2016.1203526

Calderero Hernández, J. F.; Aguirre Ocaña, A. M.; Castellanos Sánchez, A.; Peris Sirvent, R. M. \& Perochena González, P. (2014). Una nueva aproximación al concepto de educación personalizada y su relación con las TIC. Teoría de la Educación. Educación y Cultura en la Sociedad de la Información, 15(2),131-151. Recuperado de: https://www.redalyc.org/articulo.oa? id $=2010 / 201031409007$

Castells, M. (2000a) Internet y la sociedad red. Conferencia ignagural del programa de doctorado de la sociedad y el conocimiento y la información de la Universitat Oberta Catalunya. Barcelona. Recuperado de: http://www.uoc.es/web/esp/arti-cles/castells/print.html

Castells, M. (2000b) Toward a sociology of the network society. Contemporary Sociology, 29 (5), 693-699. DOI: http://dx.doi.org/10.2307/2655234

Castillo Rodríguez, C. \& Arias Montañana, B. (2018) Analysing English online resources for children: A practical Case with an evaluation template proposal. Píxel-Bit. Revista de Medio y Educación, 53, 7-25. DOI: http://dx.doi.org/10.12795/pixelbit.2018.i53.01

Castillo Rodríguez, C. \& Torrado Cespón, M. (2020) Follow the path: A learning proposal for the EFL flipped classroom. Humanidades Digitais, 2 (1).

Council of Europe. (2018) Common European Framework of Reference for Language: Learning, Teaching, Assessment. Companion Volume with New Descriptors. Strasbourg: Council of Europe. Recuperado de: https://cutt.ly/ZsdbCZO

Dewan, S. \& Riggins, F.J., 2005. The digital divide: current and future research directions. Journal of the Association for Information Systems, 6 (12), 298-337. Recuperado de: https:// cutt.ly/8sdnVZH

Dickey, M. (2004). The impact of web-logs (blogs) on student perceptions of isolation and alienation in a web-based distance-learning environment. Open Learning: The Journal of Open, Distance and e-Learning, 19 (3), 279-291. https://doi.org/ $10.1080 / 0268051042000280138$

Dörnyei, Z. \& Ushioda, E. (2011). Teaching and Researching Motivation. Harlow: Pearson. Recuperado de: https://cutt.ly/8sdmdUR

Escudero, J.M., Martínez-Domínguez, B., \& Nieto, J.M. (2018). Las TIC en la formación continua del profesorado en el contexto español. Revista de Educación, 382, 57-78. DOI https://doi.org/ 10.4438/1988-592X-RE-2018-382-392

Gardner, H. \& Davis, K. (2013) The app generation. How today's youth navigate identity, intimacy, and imagination in a digital world. New Haven: Yale University Press. Recuperado de: https://cutt.ly/OsdmLv2

Gómez González, M. A.; Sánchez Roura, M. T.; Torrado Cespón, M.; Rollings, A. \& Gómez Penas, M. D. (2016). English pronunciation for speakers of Spanish multimedia lab. http:// www.usc.es/multimlab/

Mathiasen, H. \& Schrum, L. (2010) New technologies, learning systems, and communication: Reducing complexity in the Educational System. En M. S. Knine \& I. M. Saleh (Eds.) New Science of Learning. Cognition, Computters and Collaboration in Education (pp. 385-405). London: Springer. Recuperado de: https://cutt.ly/ssdQoyq 
Mendoza Ruano, J. J. \& Caldera Serrano, J. (2014) Umbrales para la determinación de la brecha digital: comparativa entre regiones desarrolladas. Transinformaçao, 26 (2), 125-132. DOI: https://doi.org/10.1590/0103-37862014000200002

MINECO (2019) Datos particularizados de cobertura de banda ancha en Galicia a 30 de junio de 2018. Recuperado de: https://avancedigital.gob.es/banda-ancha/cobertura/ Datos\%202018/Galicia_2018.pdf

Mosteiro, M. (09/02/2020) Comienza la instalación de fibra óptica en calles del casco histórico. La Voz de Galicia. Recuperado de: https://www.lavozdegalicia.es/noticia/santiago/ 2020/02/09/comienza-instalacion-fibra-optica-calles-casco-historico/0003_202002S9C19910.htm

Nemer, D. (2015). From digital divide to digital inclusion and beyond. The Journal of Community Informatics,11 (1). http://ci-journal.net/index.php/ciej/article/view/1030

Nicolini, M. B. (2008). Chatting with letters: Developing empathy and critical literacy through writing communities. The English Journal, 97, 76-80. DOI: https://doi.org/10.2307/30046888

Pallof, R., \& Pratt, K. (2003). The virtual student. A profile and guide to working with online learners. San Francisco: Jossey Bass Wiley. Recuperado de: https://cutt.ly/nsdWdoq

Peral Peral, B.; Areas Gaitán, J \& Villarejo Ramon, A. F. (2015) De la brecha digital a la brecha psico-digital: Mayores y redes sociales. Comunicar, 45, 57-64. DOI: http://dx.doi.org/ 10.3916/C45-2015-06

Prensky, M. (2001). Digital natives, digital immigrants, Part 1. On the Horizon, 9 (5), 1-6. DOI: https://doi.org/10.1108/10748120110424816

Rasheed, A. R.; Kamsin, A.\& Abdullah, N. A. (2020) Challenges in the online component of blended learning: a systematic review. Computers \& Education, 144, 1-17. https://doi.org/10.1016/ j.compedu.2019.103701

Romero-García, C., Buzón-García, O., \& Touron, J. (2018) The flipped learning model in online education for secondary teachers. Journal of Technology and Science Education, 9(2), 109-121.DOI: https://doi.org/10.3926/jotse.435

Scollon, R. and Wong-Scollon, S. (2001) Discourse and Intercultural Communication. En D. Schiffrin, S. Tannern and H. E. Hamilton (Eds.) The Handbook of Discourse Analysis (pp. 538-547). Massachusetts: Blackwell. Recuperado de: https://cutt.ly/RsdWFOD

Sparks, C. (2013) What is the "Digital Divide" and why is it Important? Javnost - The Public: Journal of the European Institute for Communication and Culture, 20 (2), 27-46. DOI: http:// dx.doi.org/10.1080/13183222.2013.11009113

Tapscott, D. (2009). Grown up digital. How the net generation is changing your world. New York: McGraw-Hill. Recuperado de: https://cutt.ly/FsdEvHe

Tichavsky, L.P., Hunt, A.N., Driscoll, A., Jicha, K. (2015). "It's just nice having a real teacher": Student perceptions of online versus face-to-face instruction. International Journal for the Scholarship of Teaching and Learning, 9(2), 2. https://doi.org/10.20429/ijsotl.2015.090202

Torrado Cespón, M. (2015) Uso del Smartphone y su reflejo en la escritura entre estudiantes de secundaria bilingües gallego - español. Digital Education Review, 28, 77-90. DOI: https:// doi.org/10.1344/der.2015.28.77-90

Tourón, J., \& Santiago, R. (2015). El modelo flipped learning y el desarrollo del talento en la escuela. Revista de Educación, 368, 196-208. Recuperado de: https://cutt.ly/wsdRbK9 
Tourón, J., Martín, D., Navarro, E., Pradas, S.,\& Íñigo, V. (2018). Construct validation of a questionnaire to measure teachers' digital competence (TDC). Revista Española de Pedagogía, 76 (269), 25-54. DOI 10.22550/REP76-1-2018-02

Turner, A. (2015) Generation Z: Technology and social interest. The Journal of Individual Psychology, 71 (2), 103-113. DOI: https://doi.org/10.1353/jip.2015.0021

Van Deursen, A. (2007). Where to go in the near future: Diverging perspectives on online public service delivery. Lecture Notes in Computer Science, 4656, 143-154. DOI: https://doi.org/ 10.1007/978-3-540-74444-3_13

Van Deursen, A.J.A.M. and Van Dijk, J.A.G.M., 2009. Improving digital skills for the use of online public information and services. Government Information Quarterly, 26 (2), 333-340. DOI: https://doi.org/10.1016/j.giq.2008.11.002 